J Am Chem Soc. 2016 September 21; 138(37): 12021-12024. doi:10.1021/jacs.6b06566.

\title{
Hydrogen Bonding of 1,2-Azaborines in the Binding Cavity of T4 Lysozyme Mutants: Structures and Thermodynamics
}

\author{
Hyelee Lee ${ }^{\dagger}$, Marcus Fischer ${ }^{\ddagger}$, Brian K. Shoichet ${ }^{\ddagger}$, and Shih-Yuan Liu ${ }^{\dagger},{ }^{*}$ \\ tDepartment of Chemistry, Boston College, Chestnut Hill, Massachusetts 02467, United States \\ ¥Department of Pharmaceutical Chemistry, University of California, San Francisco, San Francisco, \\ California 94158, United States
}

\begin{abstract}
Protein crystallography and calorimetry were used to characterize the binding of 1,2-azaborines to model cavities in T4 lysozyme in direct comparison to their carbonaceous counterparts. In the apolar L99A cavity, affinity for Ab dropped only slightly versus benzene. In the cavity designed to accommodate a single hydrogen bond (L99A/M102Q), Gln102 $=\mathrm{O} \cdots \mathrm{H}-\mathrm{N}$ hydrogen bonding for $\mathbf{A b}$ and BEtAb was observed in the crystallographic complexes. The strength of the hydrogen bonding was estimated as 0.94 and $0.64 \mathrm{kcal} / \mathrm{mol}$ for $\mathbf{A b}$ and $\mathbf{B E t A b}$, respectively. This work unambiguously demonstrates that 1,2-azaborines can be readily accommodated in classic aryl recognition pockets and establishes one of 1,2-azaborine's distinguishing features from its carbonaceous isostere benzene: its ability to serve as an NH hydrogen bond donor in a biological setting.
\end{abstract}

The strategy of BN/CC isosterism ${ }^{1-3}$ (i.e., the replacement of a carbon-carbon (CC) unit with a boron-nitrogen (BN) unit) has emerged as a method to increase the chemical space of compounds relevant to biomedical research. ${ }^{4,5}$ When applied to a "privileged" structural motif in medicinal chemistry, this approach can produce a new versatile pharmacophore. Aromatic rings are ubiquitous in medicinal chemistry, ${ }^{6}$ and arene-containing compounds prevail among top-selling small-molecule drugs. ${ }^{7} \mathrm{BN} / \mathrm{CC}$ isosterism of arenes results in socalled azaborine heterocycles where specifically 1,2-azaborines are designated as compounds with the boron and nitrogen atoms adjacent to each other. It has been demonstrated that 1,2-azaborines can bind to a biological macromolecule, ${ }^{8}$ and that both the $B$ - and $N$-Et BN isosteres of ethylbenzene are inhibitors of ethylbenzene dehydrogenase $(\mathrm{EbDH})$, in contrast to ethylbenzene itself, which is the naturally evolved substrate for the EbDH. ${ }^{9}$ More recently, BN isosteres of naphthalene have been profiled in vitro and in vivo in terms of biological activity and ADME (absorption, distribution, metabolism, excretion) properties. ${ }^{10,11}$

\footnotetext{
*Corresponding Author. shihyuan.liu@bc.edu.

ASSOCIATED CONTENT

Supporting Information

The Supporting Information is available free of charge on the ACS Publications website at DOI: 10.1021/jacs.6b06566.

Details for synthesis of 1,2-azaborines, protein crystallization, ITC experiments, and X-ray data (PDF)

The authors declare no competing financial interest.
} 
The parental compound of the 1,2-azaborine family, 1,2-dihydro-1,2-azaborine (Ab), ${ }^{12}$ is the $\mathrm{BN}$ isostere of benzene (Figure 1). Even though 1,2-dihydro-1,2-azaborine and benzene share the same number of valence electrons, same total number of atoms, and a very similar shape, their electronic structures differ due to the presence of the polar BN bond unit in $\mathbf{A b}$ (Figure 1). For example, DFT calculations predict a dipole moment of 1,2-dihydro-1,2azaborine to be $\sim 2.2 \mathrm{D}$ vs benzene's dipole moment of 0D. ${ }^{13}$ We have also shown through deuterium labeling studies ${ }^{12}$ and small-molecule single-crystal X-ray structure analysis ${ }^{14}$ that the $\mathrm{N}-\mathrm{H}$ proton in $\mathbf{A b}$ is protic and can engage in hydrogen bonding, respectively.

Despite the recent advances in the synthetic development of 1,2-azaborines, ${ }^{15-19}$ little progress has been made in the chemistry of these BN heterocycles in a biological context. To establish 1,2-azaborines as a new pharmacophore, a fundamental understanding of the binding interactions of 1,2-azaborines with biological systems is necessary. To our knowledge, a systematic quantitative study has not appeared, and so key questions as to the effect of BN/CC isosterism on recognition in aryl binding pockets and on the hydrogenbonding potential of 1,2-azaborines have not been explored. Aryl groups in ligands are often complemented by a combination of apolar protein side chains, such as leucine, quadrupolar side chains, such as tryptophan, and occasionally even by charge-quadrupole interactions, as conferred by arginines, ${ }^{20}$ and how these interactions might be affected by 1,2 -azaborine ligands is uncertain. Similarly, hydrogen bonding plays a key role in providing directionality and specificity in protein-ligand interactions. ${ }^{21,22}$ Hydrogen bonds are widely accepted as crucial in drug-receptor binding ${ }^{23-26}$ and in maintaining favorable physical properties, and they are often installed in the course of a drug design campaign. ${ }^{27-29}$ Whether the $\mathrm{NH}$ of the 1,2-azaborine can participate in hydrogen bonds, and how these compare quantitatively to more familiar ligand-protein hydrogen bonds, also remains unexplored.

Here we combine structure and calorimetry to investigate quantitatively 1,2-azaborine's ability to replace isosteric aryl groups in arene recognition pockets and to elucidate the fundamental hydrogen bonding features of the $\mathrm{N}-\mathrm{H}$ group in 1,2-azaborines with proteins. We do so in two engineered cavity sites of T4 phage lysozyme, L99A and L99A/M102Q (described below). The quantitative structural and thermodynamic binding data that emerge will serve as general reference for future biomedical work on 1,2-azaborines.

To evaluate the possibility of hydrogen bond formation between azaborines and biological molecules, we chose cavity-bearing T4 lysozyme mutants developed by Matthews as a model system. ${ }^{30}$ Leucine to alanine mutation at the 99 position of the wild-type T4 lysozyme creates a $150 \AA^{3}$ hydrophobic pocket in the protein's C-domain. ${ }^{31,32}$ Additional mutation methionine to glutamine at the 102 position provides a more polar engineered cavity site. ${ }^{33}$ These cavities are structurally rigid, buried from bulk solvent, ${ }^{34}$ and thus are widely used as model systems to study small organic molecule (in particular arenes) binding. ${ }^{35-39}$ Additionally, their high crystallinity provides an excellent environment for unambiguous structural assignments. In other words, T4 lysozyme mutants serve as ideal biological macromolecules for a systematic physical organic structure-activity investigation. In this study we chose the L99A mutant as a nonpolar binding model and the L99A/M102Q mutant as a polar binding model with a set of four ligands (Figure 2) that include 1,2azaborines capable of $\mathrm{N}-\mathrm{H}$ hydrogen bonding (Ab, BEtAb) and control ligands that are 
incapable of N-H hydrogen bonding (Bnz, EtBnz). We investigate the binding of the two T4 lysozyme mutants L99A and L99A/M102Q with the four ligands Bnz, EtBnz, Ab, and BEtAb using high-resolution protein X-ray crystallography and isothermal titration calorimetry (ITC), and we detail our results and analysis in the following sections.

In the nonpolar cavity (L99A), X-ray crystallographic analysis of the protein-ligand complexes revealed that 1,2-azaborines and their carbonaceous counterparts bind similarly ${ }^{8}$ (Figure 3, top row). In complexes with ethyl-substituted ligands, two conformations are observed (also seen in the complexes EtBnz@L99A/M102Q and BEtAb@L99A/M102Q), and the ethyl substituent is positioned in the bulge region of the cavity, presumably due to geometric complementarity. Considering the interatomic distances (Figure 3, top row), there is no indication of the ligands forming hydrogen bonds with any of the protein residues inside the L99A binding pocket.

For the polar cavity (L99A/M102Q), it has been shown that 2-mercaptoethanol (from the crystallization buffer) and a water molecule reside in the binding pocket of the T4 lysozyme double mutant in the absence of an added ligand (PDB code: 1 LGU) ${ }^{33}$ As can be seen from Figure 3 (bottom) an arene/1,2-azaborine ligand can displace the 2-mercaptoethanol and/or water molecule from the polar binding pocket. Our structural data from the L99A/M102Q complex with $\mathbf{A b}$ show that the $\mathbf{N H}$ group of $\mathbf{A b}$ is in close proximity to carbonyl oxygen of the Gln102 residue, forming a hydrogen bond (3.1 and $3.2 \AA$ for each of the protein conformations, Figure 3 Ab@L99A/M102Q). This is the first structural characterization of an apparent hydrogen bond between an azaborine and a biological macromolecule. A distinctly different protein-ligand interaction is observed in the benzene-bound complex (Figure 3 Bnz@L99A/M102Q). A water molecule remains bound via H-bonding with Gln102 in the cavity ( 2.5 and $2.7 \AA$ from water oxygen to the carbonyl oxygen of Gln102 in the respective two conformations) along with benzene, which has no apparent bonding interaction with Gln102 (Gln102=O $\cdots$ Bnz distance $>3.5 \AA$ ). For the ethylbenzene complex EtBnz@L99A/M102Q (Figure 3), the shortest bond distance between the aromatic carbon of ethylbenzene and the carbonyl oxygen of the Gln102 is $4.6 \AA$, too far for a hydrogen bond. Conversely, what is likely a hydrogen bond, certainly a polar interaction, is observed in the BEtAb@L99A/M102Q complex (Figure 3). The observed distance between the NH group of the BEtAb ligand and the Gln102 carbonyl oxygen is $3.2 \AA$ ( $3.6 \AA$ for the second side chain conformation) in the crystal structure, which we determined to $1.47 \AA$ resolution; an amide $=\mathrm{O} \cdots \mathrm{HN}$ hydrogen bond has an average distance of $2.9 \AA \AA^{40,41}$ Furthermore, we found that the binding of Ab or BEtAb to L99A/M102Q mutant led to a movement of the Gln102 side chain toward the 1,2-azaborine substrates for the conformations that display hydrogen bonding, resulting in 0.4 and $1.1 \AA$ difference in the position of the oxygen atom of the Gln102 $=\mathrm{O}$ residue vs the corresponding control structures with no apparent hydrogen bonding interactions, respectively (Bnz vs Ab, and EtBnz vs BEtAb). Thus, it appears that the Gln102 side chain undergoes considerable geometric changes upon binding of 1,2azaborines to accommodate hydrogen bonding.

To probe the affinities of the protein-ligand interactions, we investigated the binding thermodynamics of Bnz, EtBnz, Ab, and BEtAb with L99A and L99A/M102Q T4 lysozyme mutants in aqueous solution using isothermal titration calorimetry (ITC). On 
binding to the nonpolar L99A cavity, azaborines lost between 0.4 and $0.5 \mathrm{kcal} / \mathrm{mol}$ in binding free energy compared to their corresponding carbonaceous analogs (Table 1, left colunn). This is consistent with the more polar character of the 1,2-azaborine compounds relative to their carbonaceous counterparts, and hence their higher desolvation cost.

Conversely, on binding to the polar L99A/M102Q cavity Ab and BEtAb gain between 0.4 and $0.2 \mathrm{kcal} / \mathrm{mol}$ of binding free energy relative to their carbonaceous analogs benzene and ethylbenzene, respectively (Table 1 , right column). These relative affinities are consistent with the observations from crystallographic complexes, and suggest that in a site that can accept a hydrogen bond donated by the azaborine $\mathrm{NH}$, this interaction can overcome the desolvation penalty conferred by the greater polarity of this group. We note that the ethylsubstituted derivatives EtBnz and BEtAb bind more weakly to the L99A/M102Q mutant than the unsubstituted Bnz or Ab, presumably due to the higher steric demand associated with the ethyl substituent. ${ }^{36}$

When considering the energetic contributions of protein-ligand interactions, various factors come into play, e.g., hydrophobic effect, desolvation, hydrogen bonding, electrostatic interactions, etc., ${ }^{42}$ and it can be challenging to determine a specific contribution, e.g., hydrogen bonding, from a simple comparison of a pair of free energy values, e.g., the binding free energies of Bnz and Ab to the L99A/M102Q mutant. In this simple analysis, the binding free energy difference $\Delta \Delta G=\Delta G_{\mathrm{Ab} @ \mathrm{~L} 99 \mathrm{a} / \mathrm{M} 102 \mathrm{Q}}-\Delta G_{\mathbf{B n z}} @ \mathrm{~L} 99 \mathrm{a} / \mathrm{M} 102 \mathrm{Q}$ accounts for the totality of electronic structure differences between $\mathbf{B n z}$ and $\mathbf{A b}$, not just hydrogen bonding.

Thus, we used the classic thermodynamic cycle analysis ${ }^{43-46}$ to best estimate the specific $\mathrm{Gln} 102=\mathrm{O} \cdots \mathrm{H}-\mathrm{N}$ hydrogen bonding interactions from our set of experimentally determined binding free energies $\Delta G$.

Figure 4 illustrates the double mutant cycle analysis for the benzene and ethylbenzene pairs with the determined $\mathrm{Gln} 102=\mathrm{O} \cdots \mathrm{NH}$ interaction being 0.94 and $0.64 \mathrm{kcal} / \mathrm{mol}$, respectively for Ab (Figure 4a) and BEtAb (Figure 4b). ${ }^{47}$ The comparatively weaker hydrogen bond interaction for BEtAb vs Ab with the Gln102 residue is postulated to originate from additional counteracting steric penalties incurred by the ethyl substituent and is consistent with the longer average hydrogen bond distance with Gln102 residue in the BEtAb@L99A/ M102Q structure vs the Ab@L99A/M102Q structure. The hydrogen bond strength between the azaborine $\mathrm{NH}$ and the glutamine carbonyl oxygen is within a typical range of $0.5-1.5$ $\mathrm{kcal} / \mathrm{mol}^{48}$ for amide $=\mathrm{O} \cdots \mathrm{H}-\mathrm{N}$ hydrogen bonding, and for neutral hydrogen bonds in general, in aqueous environments.

In conclusion, we have described the first quantitative experimental studies of the binding of 1,2-azaborines in an aryl recognition pocket and demonstrated the hydrogen bonding capabilities of 1,2-azaborines in a protein context. The use of high-resolution protein X-ray crystallography and ITC (1) provided quantitative measures of how the increased solubility of the 1,2-azaborines traded off against nonpolar recognition in the T4 lysozyme cavities and (2) determined $\mathrm{NH} \cdots \mathrm{O}=$ amide hydrogen bonding distances and binding free energies for 1,2-azaborines in sites designed to complement apolar and polar aryl hydrocarbons. The 
observed Gln102 $=\mathrm{O} \cdots \mathrm{H}-\mathrm{N}$ hydrogen bonding distances are 3.1 and $3.2 \AA$ for $\mathbf{A b}$ and $3.2 \AA$ for BEtAb, and the contribution of that hydrogen bond to the net free energy of binding is -0.94 and $-0.64 \mathrm{kcal} / \mathrm{mol}$ for $\mathbf{A b}$ and BEtAb, respectively. This proof-of-concept study unambiguously establishes one of 1,2-azaborine's distinguishing features from benzene, i.e., its ability to serve as an $\mathrm{NH}$ hydrogen bond donor in a biological setting and provides benchmark quantitative data that will be useful for future development of azaborines as a new pharmacophore.

\section{Supplementary Material}

Refer to Web version on PubMed Central for supplementary material.

\section{Acknowledgments}

This research was supported by the National Institutes of Health NIGMS (R01-GM094541) and Boston College (to S.-Y.L), and by NIGMS GM59957 (to B.K.S.). We thank Tao He for experimental advice and George Meigs at Beamline 8.3.1, Advanced Light Source for data collection. We also thank Jiamin Gao and Mary Roberts for helpful discussion. S.-Y.L. thanks the Camille Dreyfus Teacher-Scholar Awards Program for a Teacher-Scholar award and the Humboldt Foundation for the Friedrich Wilhelm Bessel Research Award.

\section{REFERENCES}

1. Liu Z, Marder TB. Angew. Chem., Int. Ed. 2008; 47:242.

2. Bosdet MJD, Piers WE. Can. J. Chem. 2009; 87:8.

3. Campbell PG, Marwitz AJV, Liu S-Y. Angew. Chem., Int. Ed. 2012; 51:6074.

4. Zhou H-B, Nettles KW, Bruning JB, Kim Y, Joachimiak A, Sharma S, Carlson KE, Stossi F, Katzenellenbogen BS, Greene GL, Katzenellenbogen JA. Chem. Biol. 2007; 14:659. [PubMed: 17584613]

5. Ito H, Yumura K, Saigo K. Org. Lett. 2010; 12:3386. [PubMed: 20586441]

6. Roughley SD, Jordan AM. J. Med. Chem. 2011; 54:3451. [PubMed: 21504168]

7. McGrath NA, Brichacek M, Njardarson JT. J. Chem. Educ. 2010; 87:1348.

8. Liu L, Marwitz AJV, Matthews BW, Liu S-Y. Angew. Chem., Int. Ed. 2009; 48:6817.

9. Knack DH, Marshall JL, Harlow GP, Dudzik A, Szaleniec M, Liu S-Y, Heider J. Angew. Chem., Int. Ed. 2013; 52:2599.

10. Vlasceanu A, Jessing M, Kilburn JP. Bioorg. Med. Chem. 2015; 23:4453. [PubMed: 26113188]

11. Rombouts FJ, Tovar F, Austin N, Tresadern G, Trabanco AA. J. Med. Chem. 2015; 58:9287. [PubMed: 26565745]

12. Marwitz AJV, Matus MH, Zakharov LN, Dixon DA, Liu S-Y. Angew. Chem., Int. Ed. 2009; 48:973.

13. Chrostowska A, Xu S, Lamm AN, Mazière A, Weber CD, Dargelos A, Baylère P, Graciaa A, Liu S-Y. J. Am. Chem. Soc. 2012; 134:10279. [PubMed: 22616808]

14. Abbey ER, Lamm AN, Baggett AW, Zakharov LN, Liu S-Y. J. Am. Chem. Soc. 2013; 135:12908. [PubMed: 23914914]

15. Baggett AW, Vasiliu M, Li B, Dixon DA, Liu S-Y. J. Am. Chem. Soc. 2015; 137:5536. [PubMed: 25870116]

16. Brown AN, Li B, Liu S-Y. J. Am. Chem. Soc. 2015; 137:8932. [PubMed: 26148959]

17. Amani J, Molander GA. Org. Lett. 2015; 17:3624. [PubMed: 26146880]

18. Wisniewski SR, Guenther CL, Argintaru OA, Molander GA. J. Org. Chem. 2014; 79:365. [PubMed: 24328074]

19. Rudebusch GE, Zakharov LN, Liu S-Y. Angew. Chem., Int. Ed. 2013; 52:9316. 
20. Barelier S, Sterling T, O’Meara MJ, Shoichet BK. ACS Chem. Biol. 2015; 10:2772. [PubMed: 26421501]

21. Liu Z, Wang G, Li Z, Wang R. J. Chem. Theory Comput. 2008; 4:1959. [PubMed: 26620338]

22. Fersht AR, Shi J-P, Knill-Jones J, Lowe DM, Wilkinson AJ, Blow DM, Brick P, Carter P, Waye MMY, Winter G. Nature. 1985; 314:235. [PubMed: 3845322]

23. Gonzalez AZ, Li Z, Beck HP, Canon J, Chen A, Chow D, Duquette J, Eksterowicz J, Fox BM, Fu J, Huang X, Houze J, Jin L, Li Y, Ling Y, Lo M-C, Long AM, McGee LR, McIntosh J, Oliner JD, Osgood T, Rew Y, Saiki AY, Shaffer P, Wortman S, Yakowec P, Yan X, Ye Q, Yu D, Zhao X, Zhou J, Olson SH, Sun D, Medina JC. J. Med. Chem. 2014; 57:2963. [PubMed: 24601644]

24. Verner E, Katz BA, Spencer JR, Allen D, Hataye J, Hruzewicz W, Hui HC, Kolesnikov A, Li Y, Luong C, Martelli A, Radika K, Rai R, She M, Shrader W, Sprengeler PA, Trapp S, Wang J, Young WB, Mackman RL. J. Med. Chem. 2001; 44:2753. [PubMed: 11495587]

25. Wang Y, Liu Z, Brunzelle JS, Kovari IA, Dewdney TG, Reiter SJ, Kovari LC. Biochem. Biophys. Res. Commun. 2011; 412:737. [PubMed: 21871444]

26. Das K, Sarafianos SG, Clark AD Jr, Boyer PL, Hughes SH, Arnold E. J. Mol. Biol. 2007; 365:77. [PubMed: 17056061]

27. Foloppe N, Fisher LM, Howes R, Kierstan P, Potter A, Robertson AGS, Surgenor AE. J. Med. Chem. 2005; 48:4332. [PubMed: 15974586]

28. Mills JEJ, Dean PM. J. Comput.-Aided Mol. Des. 1996; 10:607. [PubMed: 9007693]

29. Toth G, Bowers SG, Truong AP, Probst G. Curr. Pharm. Des. 2007; 13:3476. [PubMed: 18220785]

30. Eriksson AE, Baase WA, Zhang X-J, Heinz DW, Blaber M, Baldwin EP, Matthews BW. Science. 1992; 255:178. [PubMed: 1553543]

31. Eriksson AE, Baase WA, Wozniak JA, Matthews BW. Nature. 1992; 355:371. [PubMed: 1731252]

32. Morton A, Baase WA, Matthews BW. Biochemistry. 1995; 34:8564. [PubMed: 7612598]

33. Wei BQ, Baase WA, Weaver LH, Matthews BW, Shoichet BK. J. Mol. Biol. 2002; 322:339. [PubMed: 12217695]

34. Morton A, Matthews BW. Biochemistry. 1995; 34:8576. [PubMed: 7612599]

35. Graves AP, Shivakumar DM, Boyce SE, Jacobson MP, Case DA, Shoichet BK. J. Mol. Biol. 2008; 377:914. [PubMed: 18280498]

36. Merski M, Fischer M, Balius TE, Eidam O, Shoichet BK. Proc. Natl. Acad. Sci. U. S. A. 2015; 112:5039. [PubMed: 25847998]

37. Eriksson AE, Baase WA, Matthews BW. J. Mol. Biol. 1993; 229:747. [PubMed: 8433369]

38. Liu L, Baase WA, Michael MM, Matthews BW. Biochemistry. 2009; 48:8842. [PubMed: 19663503]

39. Merski M, Shoichet BK. Proc. Natl. Acad. Sci. U. S. A. 2012; 109:16179. [PubMed: 22988064]

40. Bissantz C, Kuhn B, Stahl M. J. Med. Chem. 2010; 53:5061. [PubMed: 20345171]

41. Taylor R, Kennard O, Versichel W. J. Am. Chem. Soc. 1983; 105:5761.

42. Tsai, C-J.; Norel, R.; Wolfson, HJ.; Maizel, JV.; Nussinov, R. Encyclopedia of Life Science. Chichester: John Wiley \& Sons, Ltd.; 2001.

43. Perozzo R, Folkers G, Scapozza L. J. Recept. Signal Transduction Res. 2004; 24:1.

44. Cockroft SL, Hunter CA. Chem. Soc. Rev. 2007; 36:172. [PubMed: 17264921]

45. Camara-Campos A, Musumeci D, Hunter CA, Turega S. J. Am. Chem. Soc. 2009; 131:18518. [PubMed: 19947634]

46. Roth TA, Minasov G, Morandi S, Prati F, Shoichet BK. Biochemistry. 2003; 42:14483. [PubMed: 14661960]

47. It is noteworthy to mention that these estimated energetic values may also contain an additional electrostatic contribution between dipolar azaborine molecules and protein binding sites.

48. Davis AA, Teague SJ. Angew. Chem., Int. Ed. 1999; 38:736. 
$\mathrm{p} K_{\mathrm{a}}(\mathrm{B} 3 \mathrm{LYP} / \mathrm{DZVP2})$

values in $\mathrm{H}_{2} \mathrm{O}$ (ref 15)
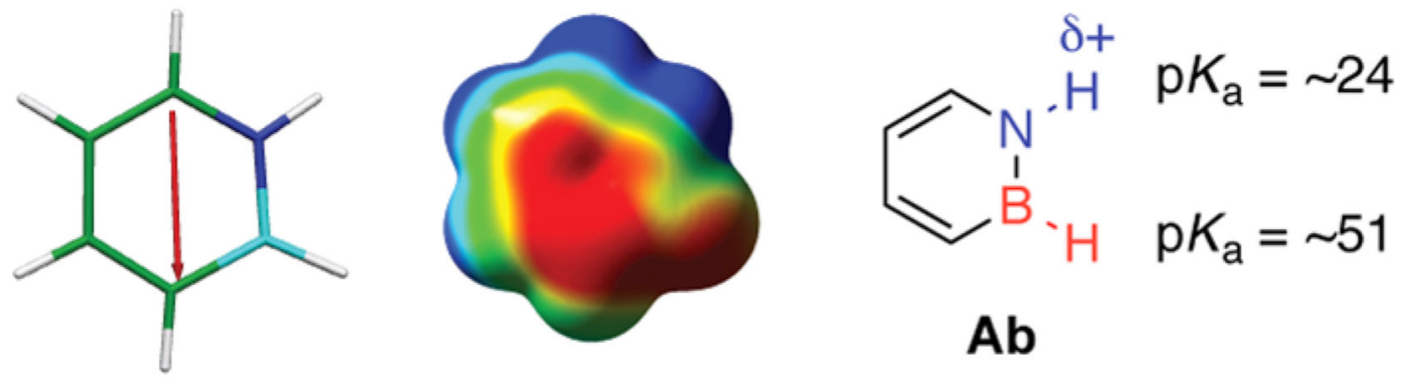

Ab

$\mathrm{N}-\mathrm{H}$ hydrogen bonding

Figure 1.

Distinguishing electronic structure features of 1,2-dihydro-1,2-azaborine as a result of $\mathrm{BN} / \mathrm{CC}$ isosterism. 
Arenes

Azaborines<smiles>c1ccccc1</smiles>
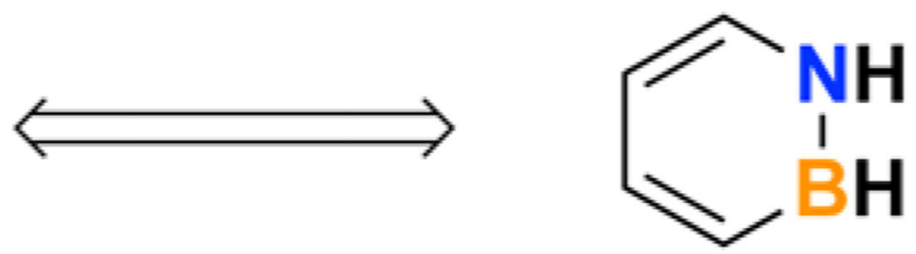

benzene

Ab (Bnz)
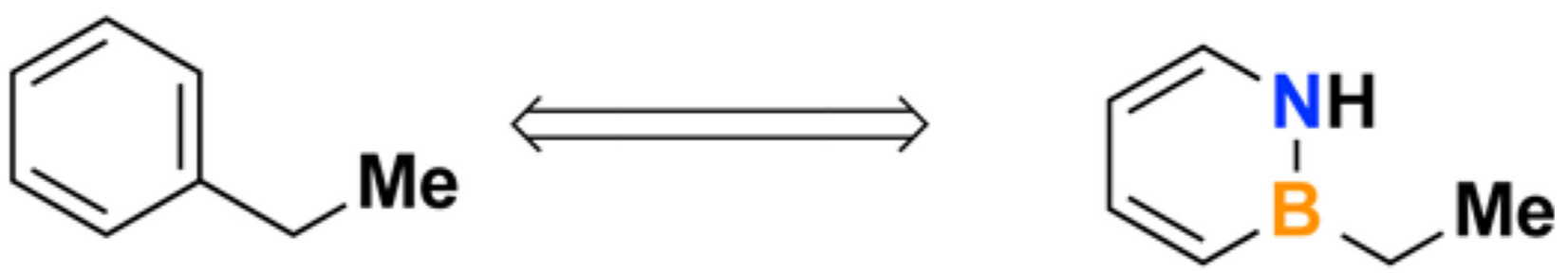

\section{ethylbenzene \\ (EtBnz)}

BEtAb

Figure 2.

Ligands examined in binding studies. Direct comparison of benzene (Bnz) versus 1,2dihydro-1,2-azaborine (Ab), and ethylbenzene (EtBnz) versus $B$-Et-1,2-azaborine (BEtAb). 


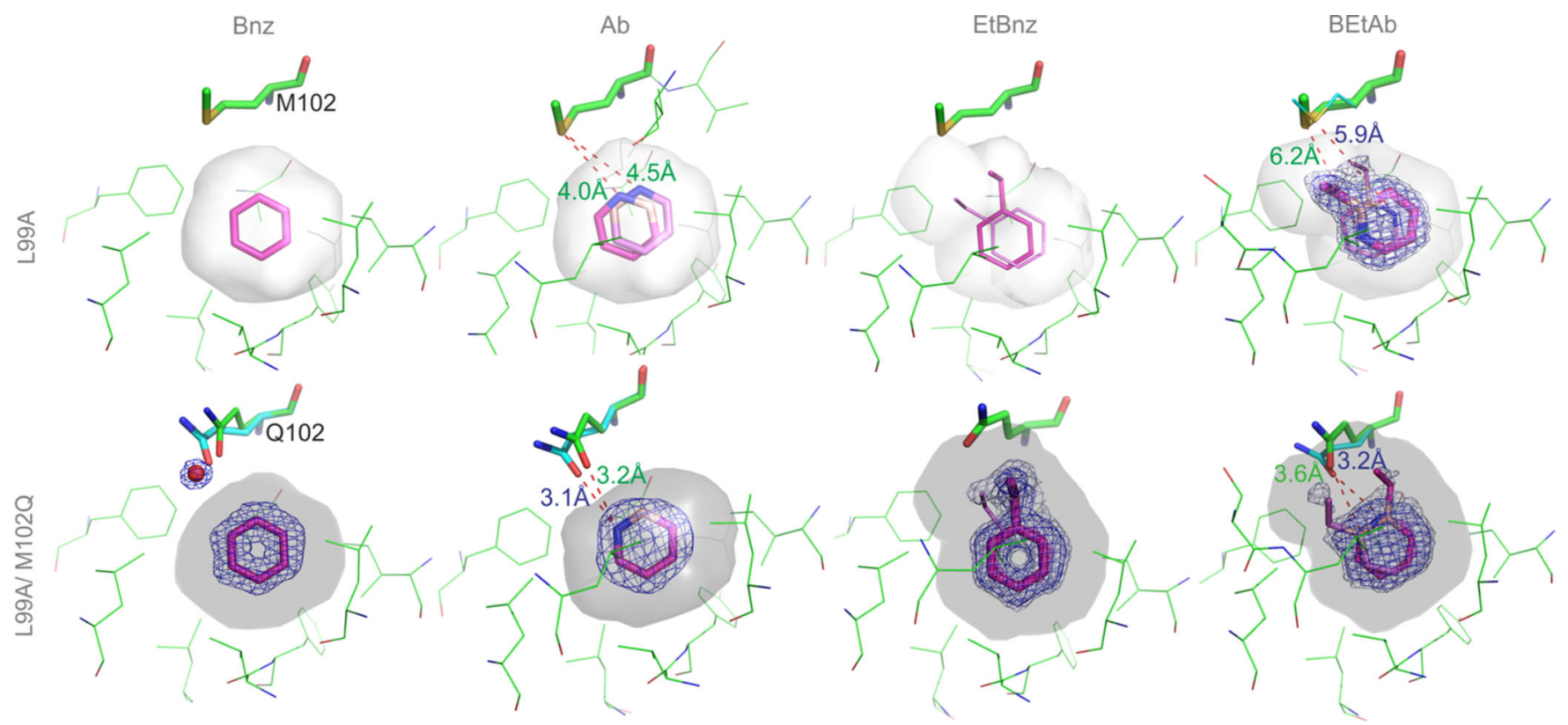

Figure 3.

Arenes and 1,2-azaborines bound in the T4 lysozyme L99A and L99A/M102Q binding pockets. Crystal structures of four ligands (purple sticks) are shown in the binding site (gray surface) of T4 lysozyme cavities L99A (top) and L99A/M102Q (bottom). Distances (in Å) between the ligand $-\mathrm{N}$ and the Met102-S or Gln102=O are illustrated in red dashes. For structures determined in this paper, electron densities are shown as blue mesh $(2 \mathrm{mFo}-\mathrm{DFc}$ map, rendered at $1 \sigma)$, and as gray mesh $(2 \mathrm{mFo}-\mathrm{DFc}, 0.5 \sigma)$ for alternative conformations of the ligand (violet sticks); stick-width reflects relative crystallographic occupancies. A water molecule in the Bnz-M102Q cavity is shown as a red sphere. 


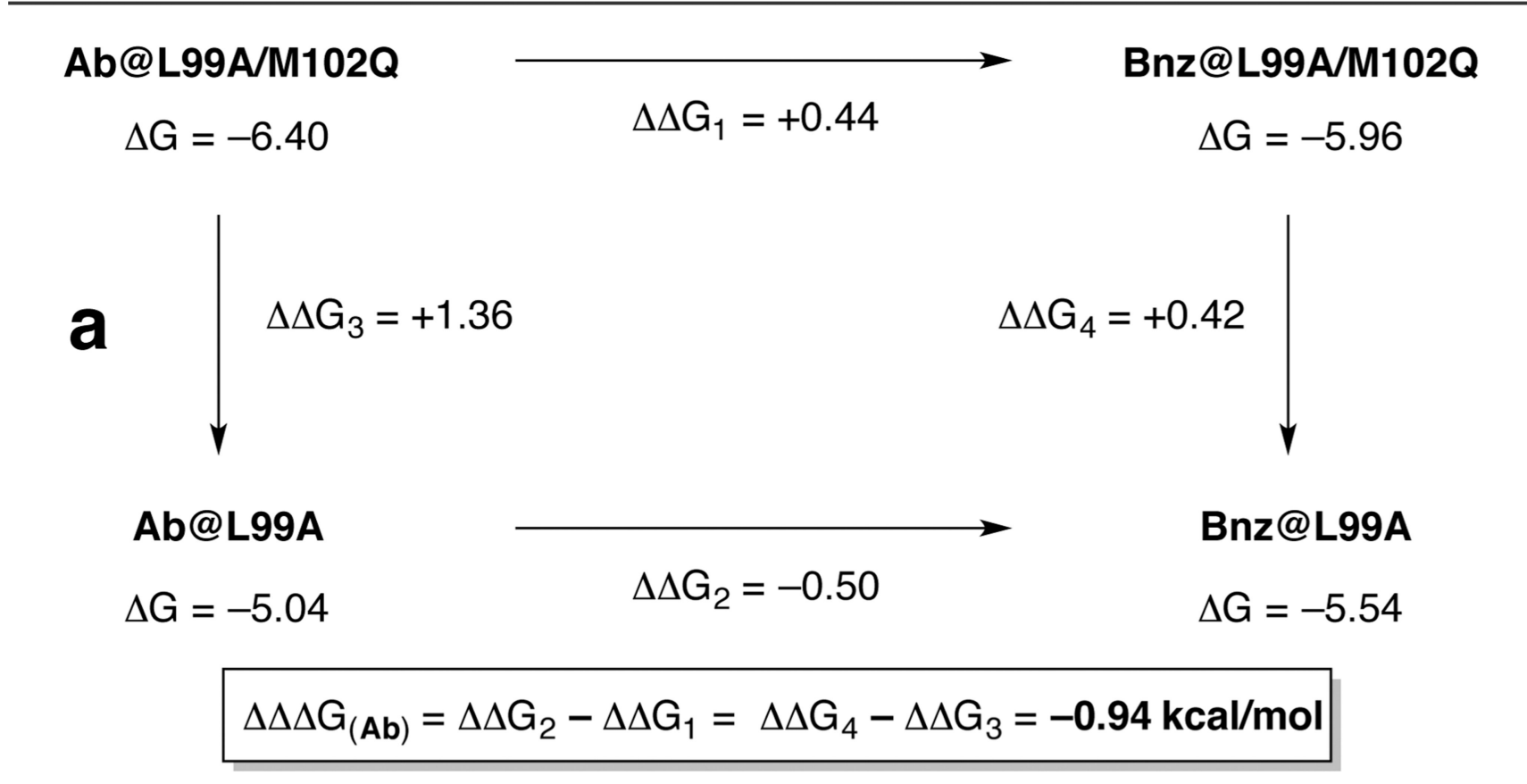

BEtAb@L99A/M102Q

$\Delta \mathrm{G}=-5.59$

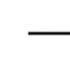

$\Delta \Delta \mathrm{G}_{5}=+0.22$

\section{EtBnz@L99A/M102Q}

$\Delta \mathrm{G}=-5.37$

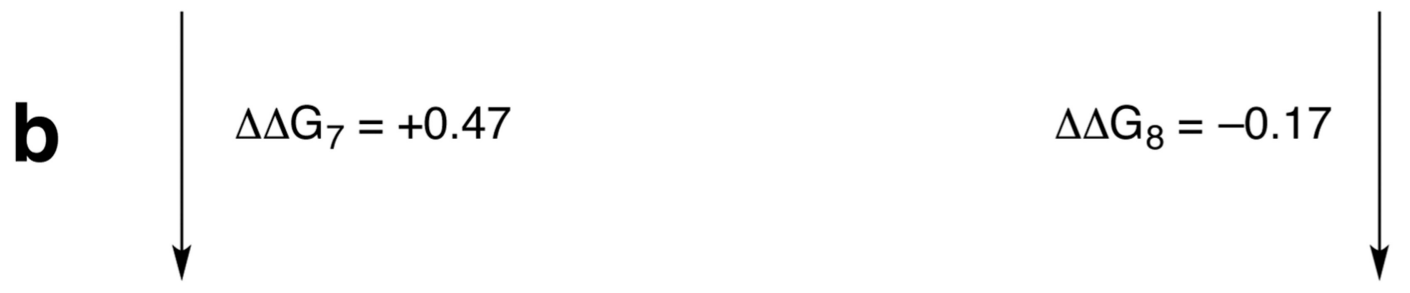

BEtAb@L99A

$\Delta G=-5.12$

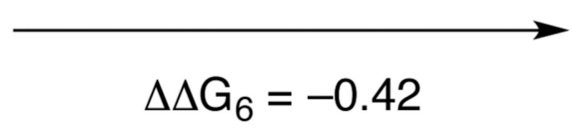

$\Delta \Delta G_{6}=-0.42$
EtBnz@L99A

$\Delta G=-5.54$

$$
\Delta \Delta \Delta G_{(B E t A b)}=\Delta \Delta G_{6}-\Delta \Delta G_{5}=\Delta \Delta G_{8}-\Delta \Delta G_{7}=-0.64 \mathrm{kcal} / \mathrm{mol}
$$

Figure 4.

Double mutant cycle analysis. (a) Free energies of hydrogen bonding between M102Q and $\mathrm{NH}$ of Ab and (b) M102Q and NH of BEtAb are estimated to be -0.94 and $-0.64 \mathrm{kcal} / \mathrm{mol}$, respectively (all values in $\mathrm{kcal} / \mathrm{mol}$ determined at $283.15 \mathrm{~K}$ ). 
Table 1

Binding Free Energy and Affinity Determined by ITC at $10{ }^{\circ} \mathrm{C}$

\begin{tabular}{lccccc}
\hline & \multicolumn{2}{c}{$\mathbf{L 9 9 A}$} & & \multicolumn{2}{c}{ L99A/M102Q } \\
\cline { 2 - 3 } \cline { 5 - 6 } & $\Delta \boldsymbol{G}(\mathbf{k c a l} / \mathbf{m o l})$ & $\boldsymbol{K}_{\mathbf{a}}\left(\times \mathbf{1 0}^{\mathbf{4}} \mathbf{M}^{-\mathbf{1}}\right)$ & & $\boldsymbol{\Delta} \boldsymbol{G}(\mathbf{k c a l} / \mathbf{m o l})$ & $\boldsymbol{K}_{\mathbf{a}}\left(\times \mathbf{1 0}^{\mathbf{4}} \mathbf{M}^{-\mathbf{1}}\right)$ \\
\hline $\mathbf{B n z}$ & $-5.54 \pm 0.04$ & $1.89 \pm 0.12$ & & $-5.96 \pm 0.04$ & $3.95 \pm 0.29$ \\
$\mathbf{A b}$ & $-5.04 \pm 0.03$ & $0.77 \pm 0.05$ & & $-6.40 \pm 0.02$ & $8.77 \pm 0.28$ \\
$\mathbf{E t B n z}$ & $-5.54 \pm 0.06$ & $1.91 \pm 0.18$ & & $-5.37 \pm 0.03$ & $1.41 \pm 0.07$ \\
$\mathbf{B E t A b}$ & $-5.12 \pm 0.02$ & $0.90 \pm 0.04$ & & $-5.59 \pm 0.02$ & $2.08 \pm 0.06$ \\
\hline
\end{tabular}

